\title{
CONHECIMENTO DOS ACADÊMICOS DE MEDICINA ACERCA DO HPV E DO CÂNCER DE COLO UTERINO
}

\author{
KNOWLEDGE OF MEDICAL STUDENTS ABOUT \\ HPV AND CERVICAL CANCER
}

\section{Samuel Laurindo da Silva', Alfredo Lustosa Vargas' ${ }^{1}$ Rogério José de Almeida ${ }^{2}$, Vera Aparecida Saddi ${ }^{3}$ Jacqueline Andréia Bernardes Leão Cordeiro', Antonio Márcio Teodoro Cordeiro Silva'}

\section{RESUMO}

O Papilomavírus humano (HPV) é capaz de induzir lesões com potencial oncogênico em tecidos epiteliais. Tendo em vista a alta prevalência mundial da infecção pelo HPV e das neoplasias associadas, questiona-se o conhecimento dos acadêmicos de Medicina sobre as características do HPV. A relevância de avaliar o conhecimento de acadêmicos de medicina sobre o tema HPV está baseada na necessidade de formar médicos com experiência sobre o assunto para que possam promover a orientação da população. Trata-se de estudo descritivo realizado com acadêmicos de Medicina da Pontifícia Universidade Católica de Goiás (Goiânia, Goiás, Brasil), por meio da aplicação de questionário validado, que avalia o nível de conhecimento sobre o HPV, a vacina e o câncer de colo uterino. A porcentagem geral de acerto foi de $80 \%$ e as médias das respostas aumentaram com 0 avanço de cada semestre. Concluiu-se, portanto, que o conhecimento foi satisfatório e progressivo.

Descritores: HPV; Neoplasias Uterinas; Vacinas contra HPV; Medicina.

\begin{abstract}
Human papillomavirus (HPV) is known to induce tissue lesions with oncogenic potential in epithelial tissues. Given the high global prevalence of HPV infection and associated neoplasia, we evaluated the knowledge of medical students about the characteristics of HPV and the effect of this virus infection in the general population's health. The importance of assessing the knowledge of medical students about the HPV is based on the necessity of training medical doctors with experince on the subject in order to promote the prevention and control new HPV cancer related cases in the population. This is a descriptive study performed with medical students at the Pontifical Catholic University of Goiás (Goiânia, Goiás, Brazil), through the application of validated questionnaire that assessed the level of knowledge about HPV infection, HPV vaccine and cervical cancer, the primary tumor associated with the virus. The overall percentage of correct answers obtained from the students was $80 \%$. By evaluating the average of responses along the development of each semester in the course. It was concluded that there was significant progression in the HPV knowledge.
\end{abstract}

Descriptors: HPV; Uterine Neoplasms; Papillomavirus Vaccines; Medicine.
${ }^{1}$ Graduado em Medicina pela Pontifícia Universidade Católica de Goiás (PUC Goiás), Goiânia, GO, Brasil.

${ }^{2}$ Doutor em Sociologia, Pósdoutorando em Ciências da Saúde, Professor Adjunto do Curso de Medicina e do Programa de PósGraduação em Ciências Ambientais e Saúde da Pontifícia Universidade Católica de Goiás (PUC Goiás), Goiânia, GO, Brasil.

${ }^{3}$ Doutora em Fisiologia Humana. Professora do Curso de Medicina e do Programa de Pós-Graduação em Ciências Ambientais e Saúde da Pontifícia Universidade Católica de Goiás (PUC Goiás), Goiânia, $\mathrm{GO}$, Brasil.

${ }^{4}$ Doutora em Enfermagem. Professora da Faculdade de Enfermagem da Universidade Federal de Goiás (PUC Goiás), Goiânia, GO, Brasil.

${ }^{5}$ Doutor em Biologia Celular e Molecular. Professor do Curso de Medicina e do Programa de PósGraduação em Ciências Ambientais e Saúde da Pontifícia Universidade Católica de Goiás (PUC Goiás), Goiânia, GO, Brasil. 


\section{Introdução}

O Papilomavírus humano (HPV) é responsável pela formação de diversas lesões tissulares epiteliais associadas ao desenvolvimento de câncer, como de colo uterino, câncer anal, cavidade oral, dentre outros ${ }^{1}$. Os HPVs são vírus epiteliotrópicos extremamente difundidos no ambiente, fato que explica a infecção cutânea e/ou mucosa, respondendo por várias neoplasias epiteliais ${ }^{2}$. Atualmente, mais de 200 tipos de HPV já foram descritos, sendo os subtipos 16 e 18 os mais associados aos cânceres causados por este vírus, especialmente, o câncer de colo de útero³.

Segundo a Organização Mundial da Saúde (OMS), aproximadamente 291 milhões de mulheres no mundo são portadoras do HPV, sendo que 32\% estão infectadas pelos tipos 16, 18 ou ambos, e estes tipos virais (16 e 18) são responsáveis por $70 \%$ dos casos de câncer de colo do útero ${ }^{4}$. Embora pouco conhecido pela população brasileira, a infecção pelo HPV se destaca como uma das doenças sexualmente transmissíveis (DST) mais comuns no mundo e uma em cada cinco mulheres é portadora do vírus ${ }^{5}$. Estudos no mundo comprovam que $80 \%$ das mulheres sexualmente ativas serão infectadas por um ou mais tipos de HPV em algum momento de suas vidas ${ }^{6}$.

$\mathrm{O}$ câncer de colo uterino, um grande problema de saúde pública mundial, associado à infecção pelo HPV7,8, corresponde, aproximadamente, a $10 \%$ de todos os casos de tumores malignos em mulheres no mundo e à segunda causa mais comum de morte por neoplasia, depois do câncer de mama. Atualmente, o HPV tem se revelado um importante problema de saúde pública especialmente em países menos desenvolvidos, nos quais ocorrem cerca de $80 \%$ dos casos de câncer de cervical ${ }^{5}$. A cada ano, ocorrem cerca de 500 mil casos de câncer de colo uterino no mundo, resultando em 270 mil mortes. No Brasil, ocorrem cerca de 20.000 casos e 4.000 mortes por ano, com um risco estimado médio de 19/100.000 mulheres. Um levantamento publicado em 2013, pelo Instituto Nacional de Câncer (INCA), mostrou que existem cerca de 18 mil novos casos de câncer de útero a cada ano no país 5 .

O uso das vacinas contra o HPV pode ser de aplicação profilática, impedindo que haja a infecção e, por essa razão, as doenças associadas. Adicionalmente, a vacina pode ser também terapêutica, devido à sua capacidade de induzir a regressão de lesões precursoras do câncer ${ }^{9,10}$. As vacinas profiláticas, atualmente, têm sido usadas em diversas partes do mundo, inclusive no Brasi $i^{9,10}$.

Neste contexto, é importante que médicos tenham informações suficientes sobre o HPV, sua comprovada associação com neoplasias ${ }^{11,12,13,14} \mathrm{e}$ a vacinação contra o vírus, tendo em vista o papel importante que desempenham na orientação da população e no esclarecimento de dúvidas aos pacientes ${ }^{1}$. Diversos estudos avaliaram o conhecimento de acadêmicos de diversos cursos no que diz respeito ao HPV e sua transmissão $0^{15,16}$.

A presente pesquisa, portanto, pode servir de parâmetro como análise situacional do nível de conhecimento relacionado ao HPV e aos cânceres associados ao vírus e pode sugerir pontos em que há dificuldades por parte dos acadêmicos, além de confirmar, como encontrado em outros estudos, diferenças relativas ao tempo de curso, gênero e situação vacinal ${ }^{17}$.

Tendo em vista às altas prevalências de cânceres associados à infecção pelo HPV questiona-se o real domínio sobre 0 conhecimento das características do vírus, das doenças associadas e os meios de prevenção por parte de acadêmicos de Medicina. Indaga-se se há uma progressão desse conhecimento com os anos na faculdade de Medicina, se a universidade realmente tem um papel de ampliar esses conhecimentos além do senso comum e se os egressos de Medicina estão com um nível de conhecimento satisfatório acerca do tema frente à situação epidemiológica dos cânceres associados ao HPV e as políticas do governo na tentativa da sua erradicação.

Este estudo tem uma dupla utilidade: trazer informações para a melhoria do ensino médico no tocante ao HPV e definir pontos que merecem destaque na orientação mais eficaz à população. Portanto, tem como objetivo avaliar os conhecimentos acerca do vírus HPV, o câncer de colo uterino e a vacina contra o HPV entre acadêmicos de Medicina da PUC-Goiás.

\section{Metodologia}

Trata-se de um estudo descritivo com 332 acadêmicos de Medicina da Pontifícia Universidade Católica de Goiás (PUCGoiás). Sendo que a coleta, o registro e a interpretação dos dados, não tiveram interferência do pesquisador ${ }^{18}$. 0 grupo amostral foi definido de acordo com os seguintes critérios de inclusão: estar devidamente matriculado no curso de Medicina da referida instituição, estar presente no ato da entrega do questionário e aceitar participar do estudo, após explicação minuciosa dos objetivos da pesquisa e assinatura do termo de consentimento livre e esclarecido. Esta pesquisa foi submetida ao Comitê de Ética em Pesquisa da PUC-Goiás e recebeu sua aprovação (CAAE: 32381114.4.0000.0037). 
Os dados foram coletados por meio de questionário previamente validado por aplicações-teste, contendo 38 questões de múltipla escolha. As 38 questões foram distribuídas da seguinte forma: cinco objetivavam a caracterização amostral, 13 avaliavam o conhecimento sobre HPV, 15 testavam o conhecimento sobre câncer de colo uterino e as cinco restantes sobre a vacina anti-HPV. Os temas abordados no questionário incluíram definições, modos de transmissão e de prevenção do contágio pelo vírus, administração e uso da vacina, riscos de desenvolvimento de câncer, dentre outros temas.

A aplicação dos questionários foi realizada antes do início de aulas, em cada turma do curso, e os alunos tiveram tempo hábil para responder o questionário sem que se identificassem. Os questionários respondidos foram entregues dentro de envelopes e devolvidos da mesma forma, sendo que os envelopes foram devolvidos lacrados para evitar a identificação do aluno. Com o questionário foi entregue o Termo de Consentimento Livre e Esclarecido, com as informações relativas à pesquisa, que foi devidamente assinado por cada participante.

Os dados da amostra foram organizados em planilhas eletrônicas no Exce ${ }^{\circledR} 2013$ (MicrosoftWindows, EUA), transportados para o programa BioEstat ${ }^{\circledR} 3.1$ e analisados por métodos de estatística descritiva e como estratégia de comparação foi utilizado o teste do qui-quadrado (x2) com intervalo de confiança de 95\%, com valor p significativo inferior a 0,05.

\section{Resultados e Discussão}

A partir dos dados coletados dentre os acadêmicos participantes da pesquisa ( $n=332$; taxa de participação de $60,9 \%$ em relação ao total de alunos matriculados no curso), pôde-se traçar um perfil característico da população. Dentre estes, a maioria era do sexo feminino $(64,2 \%)$, com idade entre 18 e 23 anos $(72,6 \%)$, solteiro $(93,1 \%$; $p=0,0003)$ e declarando-se com vida sexual ativa $(57,5 \%)$. Cerca de $15,4 \%(n=51)$ não informaram no questionário se tinham vida sexual ativa (Tabela 1). Adicionalmente, os dados foram estratificados em conhecimento sobre o HPV, sobre o câncer de colo uterino e a vacina anti-HPV.

Tabela 1 - Informações gerais sobre os acadêmicos de Medicina da PUC Goiás,

\begin{tabular}{|c|c|c|c|c|c|c|}
\hline \multirow[t]{2}{*}{ Parâmetros } & \multicolumn{2}{|c|}{$\begin{array}{l}\text { Feminino } \\
(n=213)\end{array}$} & \multicolumn{2}{|c|}{$\begin{array}{l}\text { Masculino } \\
(n=119)\end{array}$} & \multicolumn{2}{|c|}{$\begin{array}{l}\text { Todos } \\
(n=332)\end{array}$} \\
\hline & n & $f(\%)$ & $\mathbf{n}$ & $f(\%)$ & $\mathbf{n}$ & $f(\%)$ \\
\hline \multicolumn{7}{|l|}{ Idade (anos) } \\
\hline 18 a 23 & 156 & 73,2 & 85 & 71,4 & 241 & 72,6 \\
\hline 24 a 29 & 53 & 24,9 & 29 & 24,4 & 82 & 24,7 \\
\hline$\geq 30$ & 4 & 1,9 & 5 & 4,2 & 9 & 2,7 \\
\hline \multicolumn{7}{|l|}{ Estado civil } \\
\hline Solteiro & 197 & 92,5 & 112 & 94,1 & 309 & 93,1 \\
\hline Casado & 9 & 4,2 & 2 & 1,7 & 11 & 3,3 \\
\hline Outro & 5 & 2,3 & 2 & 1,7 & 7 & 2,1 \\
\hline NR & 2 & 0,9 & 3 & 2,5 & 5 & 1,5 \\
\hline
\end{tabular}

Período do Curso

\begin{tabular}{c|c|c|c|c|c|c}
\hline Primeiro & 24 & 11,3 & 26 & 21,8 & 50 & 15,1 \\
\hline Segundo & 12 & 5,6 & 8 & 6,7 & 20 & 6,0 \\
\hline Terceiro & 26 & 12,2 & 7 & 5,9 & 33 & 9,9 \\
\hline Quarto & 11 & 5,2 & 11 & 9,2 & 22 & 6,6 \\
\hline Quinto & 16 & 7,5 & 7 & 5,9 & 23 & 6,9 \\
\hline Sexto & 26 & 12,2 & 14 & 11,8 & 40 & 12,0 \\
\hline Sétimo & 9 & 4,2 & 8 & 6,7 & 17 & 5,1 \\
\hline Oitavo & 23 & 10,8 & 8 & 6,7 & 31 & 9,3 \\
\hline
\end{tabular}

Continua... 


\begin{tabular}{|c|c|c|c|c|c|c|}
\hline \multirow{2}{*}{ Parâmetros } & \multicolumn{2}{|c|}{$\begin{array}{l}\text { Feminino } \\
(n=213)\end{array}$} & \multicolumn{2}{|c|}{$\begin{array}{l}\text { Masculino } \\
(n=119)\end{array}$} & \multicolumn{2}{|c|}{$\begin{array}{l}\text { Todos } \\
(n=332)\end{array}$} \\
\hline & $\mathbf{n}$ & $f(\%)$ & $\mathbf{n}$ & $f(\%)$ & $\mathbf{n}$ & $f(\%)$ \\
\hline \multicolumn{7}{|l|}{ Período do Curso } \\
\hline Nono & 13 & 6,1 & 8 & 6,7 & 21 & 6,3 \\
\hline Décimo & 22 & 10,3 & 8 & 6,7 & 30 & 9,0 \\
\hline Décimo primeiro & 18 & 8,5 & 11 & 9,2 & 29 & 8,7 \\
\hline Décimo segundo & 13 & 6,1 & 3 & 2,5 & 16 & 4,8 \\
\hline \multicolumn{7}{|l|}{ Vida Sexual Ativa } \\
\hline Sim & 107 & 50,2 & 84 & 70,6 & 191 & 57,5 \\
\hline Não & 71 & 33,3 & 19 & 16,0 & 90 & 27,1 \\
\hline NR & 35 & 16,4 & 16 & 13,4 & 51 & 15,4 \\
\hline
\end{tabular}

Em relação aos conhecimentos gerais sobre o Papilomavírus humano (HPV), a grande maioria já ouviu falar sobre o vírus (330; 99,4\%). Sobre a infecção e seus mecanismos, 95,8\% (318) dos acadêmicos a reconhecem como uma DST e grande parte tem conhecimento acerca da alta frequência da infecção pelo vírus (314; 94,6\%) e de alguns meios de transmissão como o contato com fluidos corporais (261; 78,6\%) e de mãe para filho (270; 81,3\%) (Tabela 2).

Em relação a outros estudos realizados com estudantes de medicina, verifica-se um bom desempenho no quesito contato prévio com informações sobre o HPV. Em um estudo realizado com 878 estudantes de medicina na Universidade de Chongqing (China), 76,5\% dos estudantes já tinham ouvido falar do vírus ${ }^{19}$.

Avaliando outros estudos, que expandiram o grupo de estudo para todos os estudantes universitários (independente da área), os resultados são piores, como no caso da Universidade de Pequim, com apenas $12 \%$ dos acadêmicos tendo algum conhecimento prévio sobre o HPV ${ }^{20}$. Grande parte dos entrevistados no presente estudo entende como faixa etária mais acometida a de adultos jovens de ambos os sexos $(314 ; 94,6 \%)$, com vida sexual ativa $(294 ; 88,6 \%)$ e que na maior parte dos indivíduos acometidos a infecção é assintomática (297; 89,5\%).

Tabela 2 - Conhecimento dos acadêmicos de Medicina da PUC Goiás sobre o HPV, Goiânia/GO.

\begin{tabular}{|c|c|c|c|c|c|c|c|}
\hline \multirow{2}{*}{ Parâmetros } & \multicolumn{2}{|c|}{$\begin{array}{c}\text { Feminino } \\
(n=213)\end{array}$} & \multicolumn{2}{|c|}{$\begin{array}{c}\text { Masculino } \\
\text { (n=119) }\end{array}$} & \multicolumn{2}{|c|}{$\begin{array}{l}\text { Todos } \\
(n=332)\end{array}$} & \multirow{2}{*}{ p-valor } \\
\hline & $\mathbf{n}$ & $f(\%)$ & $\mathbf{n}$ & $f(\%)$ & $\mathbf{n}$ & $f(\%)$ & \\
\hline \multicolumn{8}{|c|}{ Já ouviu falar sobre o HPV? } \\
\hline Sim & 212 & 99,5 & 118 & 99,2 & 330 & 99,4 & \multirow{2}{*}{0,748} \\
\hline Não & 1 & 0,5 & 1 & 0,8 & 2 & 0,6 & \\
\hline
\end{tabular}

A infecção pelo HPV é uma DST?

\begin{tabular}{c|c|c|c|c|c|c|c}
\hline Sim & 203 & 95,3 & 115 & 96,6 & 318 & 95,8 & \\
\cline { 1 - 6 } Não & 9 & 4,2 & 4 & 3,4 & 13 & 3,9 & 0,918 \\
\cline { 1 - 6 } NSR & 1 & 0,5 & 0 & 0,0 & 1 & 0,3 & \\
\hline
\end{tabular}

O beijo é uma forma de transmissão do HPV?

\begin{tabular}{c|c|c|c|c|c|c|c}
\hline $\operatorname{Sim}$ & 37 & 17,4 & 26 & 21,8 & 63 & 19,0 & \\
\cline { 1 - 6 } Não & 174 & 81,7 & 88 & 73,9 & 262 & 78,9 & \multirow{2}{*}{0,317} \\
\cline { 1 - 6 } NSR & 2 & 0,9 & 5 & 4,2 & 7 & 2,1 & \\
\hline
\end{tabular}

O contato direto com fluidos corporais pode transmitir HPV?

\begin{tabular}{c|c|c|c|c|c|c|c}
\hline Sim & 169 & 79,3 & 92 & 77,3 & 261 & 78,6 & \multirow{2}{*}{0,928} \\
\cline { 1 - 6 } Não & 43 & 20,2 & 25 & 21,0 & 68 & 20,5 & \multirow{2}{*}{ Continua... } \\
\cline { 1 - 6 } NSR & 1 & 0,5 & 2 & 1,7 & 3 & 0,9 & \\
\hline
\end{tabular}




\begin{tabular}{|c|c|c|c|c|c|}
\hline \multirow{2}{*}{ Parâmetros } & $\begin{array}{c}\text { Feminino } \\
(n=213)\end{array}$ & $\begin{array}{c}\text { Masculino } \\
(n=119)\end{array}$ & \multicolumn{2}{|c|}{$\begin{array}{l}\text { Todos } \\
(n=332)\end{array}$} & \multirow[t]{2}{*}{ p-valor } \\
\hline & $f(\%)$ & $f(\%)$ & $\mathbf{n}$ & $f(\%)$ & \\
\hline
\end{tabular}

Água contaminada pode transmitir HPV?

\begin{tabular}{c|c|c|c|c|c|c|c}
\hline Sim & 12 & 5,6 & 15 & 12,6 & 27 & 8,1 & \multirow{2}{*}{$0.046^{*}$} \\
\cline { 1 - 6 } Não & 199 & 93,4 & 104 & 87,4 & 303 & 91,3 & \\
\hline NSR & 2 & 0,9 & 0 & 0,0 & 2 & 0,6 & \\
\hline
\end{tabular}

Existe transmissão de HPV de mãe para filho?

\begin{tabular}{c|c|c|c|c|c|c|c}
\hline Sim & 169 & 79,3 & 101 & 84,9 & 270 & 81,3 & \\
\cline { 1 - 6 } Não & 37 & 17,4 & 18 & 15,1 & 55 & 16,6 & 0,615 \\
\cline { 1 - 5 } NSR & 7 & 3,3 & 0 & 0,0 & 7 & 2,1 & \\
\hline
\end{tabular}

A infecção pelo HPV é comum?

\begin{tabular}{c|c|c|c|c|c|c|}
\hline Sim & 204 & 95,8 & 110 & 92,4 & 314 & 94,6 \\
\hline Não & 5 & 2,3 & 9 & 7,6 & 14 & 4,2 \\
\hline NSR & 4 & 1,9 & 0 & 0,0 & 4 & 1,2 \\
\hline
\end{tabular}

A infecção pelo HPV pode causar câncer cervical?

\begin{tabular}{c|c|c|c|c|c|c|}
\hline $\operatorname{sim}$ & 188 & 88,3 & 104 & 87,4 & 292 & 88,0 \\
\hline Não & 19 & 8,9 & 14 & 11,8 & 33 & 9,9 \\
\hline NSR & 6 & 2,8 & 1 & 0,8 & 7 & 2,1 \\
\hline
\end{tabular}

\section{Quem pode se infectar pelo HPV?}

\begin{tabular}{c|c|c|c|c|c|c|}
\hline Mulheres ou Homens & 12 & 5,6 & 5 & 4,2 & 17 & 5,1 \\
\hline Ambos & 200 & 93,9 & 114 & 95,8 & 314 & 94,6 \\
\hline NSR & 1 & 0,5 & 0 & 0,0 & 1 & 0,3 \\
\hline
\end{tabular}

A incidência da infecção pelo HPV é maior em mulheres entre 20 e 30 anos?

\begin{tabular}{c|c|c|c|c|c|c|}
\hline Sim & 190 & 89,2 & 104 & 87,4 & 294 & 88,6 \\
\hline Não & 18 & 8,5 & 13 & 10,9 & 31 & 9,3 \\
\hline NSR & 5 & 2,3 & 2 & 1,7 & 7 & 2,1 \\
\hline
\end{tabular}

A infecção pelo HPV é na maioria das vezes:

\begin{tabular}{c|c|c|c|c|c|c|}
\hline Sintomática & 22 & 10,3 & 11 & 9,2 & 33 & 9,9 \\
\hline Assintomática & 189 & 88,7 & 108 & 90,8 & 297 & 89,5 \\
\hline NSR & 2 & 0,9 & 0 & 0,0 & 2 & 0,6 \\
\hline
\end{tabular}

A infecção pelo HPV causa verrugas genitais?

\begin{tabular}{c|c|c|c|c|c|c|}
\hline Sim & 204 & 95,8 & 112 & 94,1 & 316 & 95,2 \\
\hline Não & 9 & 4,2 & 5 & 4,2 & 14 & 4,2 \\
\hline NSR & 0 & 0,0 & 2 & 1,7 & 2 & 0,6 \\
\hline
\end{tabular}

A infecção pelo HPV tem cura?

\begin{tabular}{c|c|c|c|c|c|c|}
\hline $\operatorname{Sim}$ & 119 & 55,9 & 75 & 63,0 & 194 & 58,4 \\
\hline Não & 93 & 43,7 & 44 & 37,0 & 137 & 41,3 \\
\hline NSR & 1 & 0,5 & 0 & 0,0 & 1 & 0,3 \\
\hline
\end{tabular}


O reconhecimento de que a infecção pelo HPV é assintomática e que a infecção está especialmente relacionada às atividades sexuais é importante para que o médico promova melhor orientação ao paciente, mesmo àqueles que não iniciaram sua vida sexual. Avaliando esse paciente alvo, pode-se executar prevenção primária, tendo em vista que não ter experiência sexual está associado a menor conhecimento sobre o vírus entre jovens estudantes, além de uma associação com sexo masculino e ausência de plano de saúde ${ }^{21}$.

Os acadêmicos de medicina da PUC-Goiás, que participaram deste estudo, entendem o papel do HPV como fator de risco para o câncer cervical $(292 ; 88,0 \%)$ e na gênese de verrugas genitais (316; $95,2 \%)$. Por outro lado, os entrevistados têm dúvidas sobre a possibilidade de cura da infecção pelo HPV, pois parte acredita que existe chance de erradicação do vírus (194; 58,4\%), enquanto 41,3\% (137) acredita que não (Tabela 3).

Na questão acerca da associação do HPV com câncer de colo uterino, comparando com outros estudos semelhantes, o desempenho foi similar ${ }^{22}$. Os resultados dos estudantes da PUC-Goiás foram semelhantes aos estudos com acadêmicos de Medicina da Índia, com 89,2\% destes acadêmicos revelando conhecimento dessa associação de risco²2.

No que se refere ao câncer cervical e sua relação com a infecção pelo HPV, os acadêmicos entendem o papel do vírus na patogênese deste câncer (292; 88,0\%), porém, parte (76; 22,9\%) não percebe o fator genético relacionado ao câncer e alguns acreditam que infecção bacteriana pode causar o câncer (160; 48,2\%).

Tabela 3 - Conhecimento acerca do câncer de colo uterino, Goiânia/Co.

\begin{tabular}{|c|c|c|c|c|c|c|c|}
\hline \multirow{2}{*}{ Parâmetros } & \multicolumn{2}{|c|}{$\begin{array}{c}\text { Feminino } \\
(n=213)\end{array}$} & \multicolumn{2}{|c|}{$\begin{array}{c}\text { Masculino } \\
(n=119)\end{array}$} & \multicolumn{2}{|c|}{$\begin{array}{l}\text { Todos } \\
(n=332)\end{array}$} & \multirow{2}{*}{ p-valor } \\
\hline & $\mathbf{n}$ & $f(\%)$ & $\mathbf{n}$ & $f(\%)$ & $\mathbf{n}$ & $f(\%)$ & \\
\hline \multicolumn{8}{|c|}{ A infecção pelo HPV pode causar câncer cervical? } \\
\hline Sim & 191 & 89,7 & 102 & 85,7 & 293 & 88,3 & \multirow{3}{*}{0,308} \\
\hline Não & 21 & 9,9 & 17 & 14,3 & 38 & 11,4 & \\
\hline NSR & 1 & 0,5 & 0 & 0,0 & 1 & 0,3 & \\
\hline
\end{tabular}

O câncer cervical está relacionado à predisposição genética?

\begin{tabular}{|c|c|c|c|c|c|c|c|}
\hline Sim & 161 & 75,6 & 94 & 79,0 & 255 & 76,8 & \multirow{3}{*}{0,620} \\
\hline Não & 51 & 23,9 & 25 & 21,0 & 76 & 22,9 & \\
\hline NSR & 1 & 0,5 & 0 & 0,0 & 1 & 0,3 & \\
\hline
\end{tabular}

Alguns alimentos podem causar câncer cervical?

\begin{tabular}{|c|c|c|c|c|c|c|c|}
\hline Sim & 32 & 15,0 & 31 & 26,1 & 63 & 19,0 & \multirow{3}{*}{$0.017^{*}$} \\
\hline Não & 181 & 85,0 & 86 & 72,3 & 267 & 80,4 & \\
\hline NSR & 0 & 0,0 & 2 & 1,7 & 2 & 0,6 & \\
\hline
\end{tabular}

Infecção bacteriana pode causar câncer cervical?

\begin{tabular}{c|c|c|c|c|c|c|c}
\hline Sim & 101 & 47,4 & 59 & 49,6 & 160 & 48,2 & \\
\cline { 1 - 6 } Não & 105 & 49,3 & 59 & 49,6 & 164 & 49,4 & \multirow{2}{*}{0,958} \\
\cline { 1 - 5 } NSR & 7 & 3,3 & 1 & 0,8 & 8 & 2,4 & \\
\hline
\end{tabular}

Ter múltiplos parceiros sexuais é um fator de risco para o desenvolvimento do câncer cervical?

\begin{tabular}{c|c|c|c|c|c|c|c}
\hline Sim & 200 & 93,9 & 104 & 87,4 & 304 & 91,6 & \multirow{2}{*}{0,066} \\
\cline { 1 - 6 } Não & 13 & 6,1 & 15 & 12,6 & 28 & 8,4 & \\
\hline
\end{tabular}

Relação sexual precoce é caracterizada como um fator de risco para o câncer cervical?

\begin{tabular}{c|c|c|c|c|c|c|c}
\hline $\operatorname{Sim}$ & 175 & 82,2 & 88 & 73,9 & 263 & 79,2 & \\
\cline { 1 - 6 } Não & 36 & 16,9 & 30 & 25,2 & 66 & 19,9 & \multirow{2}{*}{0,094} \\
\cline { 1 - 6 } NSR & 2 & 0,9 & 1 & 0,8 & 3 & 0,9 & \\
\hline
\end{tabular}




\begin{tabular}{|c|c|c|c|c|c|c|c|}
\hline \multirow{2}{*}{ Parâmetros } & \multicolumn{2}{|c|}{$\begin{array}{c}\text { Feminino } \\
(n=213)\end{array}$} & \multicolumn{2}{|c|}{$\begin{array}{c}\text { Masculino } \\
(n=119)\end{array}$} & \multicolumn{2}{|c|}{$\begin{array}{l}\text { Todos } \\
(n=332)\end{array}$} & \multirow{2}{*}{ p-valor } \\
\hline & $\mathbf{n}$ & $f(\%)$ & $\mathbf{n}$ & $f(\%)$ & $\mathbf{n}$ & $f(\%)$ & \\
\hline
\end{tabular}

O uso de DIU (Dispositivo Intrauterino) é um fator de risco para o câncer cervical?

\begin{tabular}{c|c|c|c|c|c|c|c}
\hline Sim & 67 & 31,5 & 34 & 28,6 & 101 & 30,4 & \\
\cline { 1 - 6 } Não & 146 & 68,5 & 84 & 70,6 & 230 & 69,3 & \multirow{2}{*}{0,707} \\
\cline { 1 - 5 } NSR & 0 & 0,0 & 1 & 0,8 & 1 & 0,3 & \\
\hline
\end{tabular}

O tabagismo é um fator de risco para o desenvolvimento do câncer cervical?

\begin{tabular}{c|c|c|c|c|c|c|c}
\hline $\operatorname{sim}$ & 148 & 69,5 & 89 & 74,8 & 237 & 71,4 & \\
\cline { 1 - 6 } Não & 64 & 30,0 & 30 & 25,2 & 94 & 28,3 & \multirow{2}{*}{0,403} \\
\cline { 1 - 5 } NSR & 1 & 0,5 & 0 & 0,0 & 1 & 0,3 & \\
\hline
\end{tabular}

O etilismo é um fator de risco para o câncer cervical?

\begin{tabular}{c|c|c|c|c|c|c|c}
\hline $\operatorname{sim}$ & 103 & 48,4 & 61 & 51,3 & 164 & 49,4 & \multirow{2}{*}{0,694} \\
\cline { 1 - 6 } Não & 110 & 51,6 & 58 & 48,7 & 168 & 50,6 & \\
\hline
\end{tabular}

Falta de higiene é um fator de risco para o câncer cervical?

\begin{tabular}{c|c|c|c|c|c|c|c}
\hline Sim & 148 & 69,5 & 91 & 76,5 & 239 & 72,0 & \\
\cline { 1 - 6 } Não & 63 & 29,6 & 27 & 22,7 & 90 & 27,1 & 0,218 \\
\cline { 1 - 5 } NSR & 2 & 0,9 & 1 & 0,8 & 3 & 0,9 & \\
\hline
\end{tabular}

Dor após relação sexual é um dos sintomas do câncer cervical?

\begin{tabular}{c|c|c|c|c|c|c|}
\hline $\operatorname{sim}$ & 158 & 74,2 & 87 & 73,1 & 245 & 73,8 \\
\hline Não & 54 & 25,4 & 30 & 25,2 & 84 & 25,3 \\
\hline NSR & 1 & 0,5 & 2 & 1,7 & 3 & 0,9 \\
\hline
\end{tabular}

0,922

Sangramentos entre as menstruações são sintomas do câncer cervical?

\begin{tabular}{c|c|c|c|c|c|c|c}
\hline $\operatorname{sim}$ & 175 & 82,2 & 77 & 64,7 & 252 & 75,9 & \\
\cline { 1 - 6 } Não & 36 & 16,9 & 41 & 34,5 & 77 & 23,2 & \multirow{2}{*}{$0.0005^{*}$} \\
\cline { 1 - 5 } NSR & 2 & 0,9 & 1 & 0,8 & 3 & 0,9 & \\
\hline
\end{tabular}

A presença de corrimento sanguinolento é sintoma do câncer cervical?

\begin{tabular}{c|c|c|c|c|c|c|c}
\hline Sim & 181 & 85,0 & 85 & 71,4 & 266 & 80,1 & \\
\cline { 1 - 6 } Não & 32 & 15,0 & 33 & 27,7 & 65 & 19,6 & \multirow{2}{*}{$0.007^{*}$} \\
\cline { 1 - 5 } NSR & 0 & 0,0 & 1 & 0,8 & 1 & 0,3 & \\
\hline
\end{tabular}

Febre e dor de cabeça são sintomas do câncer cervical?

\begin{tabular}{c|c|c|c|c|c|c|c}
\hline Sim & 56 & 26,3 & 43 & 36,1 & 99 & 29,8 & \\
\cline { 1 - 6 } Não & 156 & 73,2 & 73 & 61,3 & 229 & 69,0 & 0,060 \\
\cline { 1 - 5 } NSR & 1 & 0,5 & 3 & 2,5 & 4 & 1,2 & \\
\hline
\end{tabular}

Dor pélvica é um sintoma característico do câncer cervical?

\begin{tabular}{c|c|c|c|c|c|c|}
\hline $\operatorname{Sim}$ & 151 & 70,9 & 77 & 64,7 & 228 & 68,7 \\
\hline Não & 60 & 28,2 & 41 & 34,5 & 101 & 30,4 \\
\hline NSR & 2 & 0,9 & 1 & 0,8 & 3 & 0,9 \\
\hline
\end{tabular}

Adicionalmente, os entrevistados entendem que o câncer uterino também está associado a determinados padrões comportamentais como múltiplos parceiros $(304 ; 91,6 \%)$, tabagismo $(237 ; 71,4 \%)$ e início precoce das relações sexuais 
(263; 79,2\%). Grande parte dos acadêmicos associa o câncer cervical à falta de higiene (239; 72,0\%), porém ficam divididos em relação ao etilismo com 50,6\% (168) acreditando que não se trata de fator de risco relevante.

Em relação ao reconhecimento dos sintomas, os acadêmicos identificam como queixas suspeitas, o sangramento vaginal fora do ciclo menstrual $(252 ; 75,9 \% ; p=0,0005)$, corrimento vaginal sanguinolento $(266 ; 80,1 \% ; p=0,007)$ e dor pélvica (228; 68,7\%). Todavia, algumas queixas que geralmente não são relacionadas foram apontadas como características, como febre e dores de cabeça $(99 ; 29,8 \%)$ e dispareunia $(245 ; 73,8 \%)$.

No que concerne aos conhecimentos básicos relativos à vacina do HPV, importante instrumento de controle da infecção e prevenção do câncer cervical, os acadêmicos, em sua maioria, já ouviram falar da vacina (322; 97,0\%; $p=0,022)$, entendem a importância do uso preventivo em indivíduos que não tiveram contato com o vírus (315; $94,9 \%$; $p=0,024)$ e, por isso, percebem a necessidade de vacinar a faixa etária a partir dos 10 anos $(303 ; 91,3 \%)$. Os entrevistados concordam que mesmo vacinadas as mulheres precisam realizar o exame de Papanicolau (302; 91,0\%). Porém, parte dos acadêmicos acha que a vacina só poderia ser administrada em mulheres (93; 28,0\%) (Tabela 4).

Tabela 4 - Conhecimento dos acadêmicos de Medicina da PUC Goiás sobre vacina contra o HPV, Goiânia/GO.

\begin{tabular}{|c|c|c|c|c|c|c|c|}
\hline \multirow{2}{*}{ Parâmetros } & \multicolumn{2}{|c|}{$\begin{array}{c}\text { Feminino } \\
(n=213)\end{array}$} & \multicolumn{2}{|c|}{$\begin{array}{c}\text { Masculino } \\
(n=119)\end{array}$} & \multicolumn{2}{|c|}{$\begin{array}{l}\text { Todos } \\
(n=332)\end{array}$} & \multirow{2}{*}{ p-valor } \\
\hline & $\mathbf{n}$ & $f(\%)$ & $\mathbf{n}$ & $f(\%)$ & $\mathbf{n}$ & $f(\%)$ & \\
\hline \multicolumn{8}{|c|}{ Você já ouviu falar sobre a vacina anti HPV? } \\
\hline Sim & 210 & 98,6 & 112 & 94,1 & 322 & 97,0 & \multirow{3}{*}{$0.022^{*}$} \\
\hline Não & 2 & 0,9 & 7 & 5,9 & 9 & 2,7 & \\
\hline NSR & 1 & 0,5 & 0 & 0,0 & 1 & 0,3 & \\
\hline
\end{tabular}

A vacina é aprovada para indivíduos que não tiveram contato com o vírus do HPV?

\begin{tabular}{|c|c|c|c|c|c|c|c|}
\hline Sim & 206 & 96,7 & 109 & 91,6 & 315 & 94,9 & \multirow{3}{*}{$0.024^{*}$} \\
\hline Não & 5 & 2,3 & 10 & 8,4 & 15 & 4,5 & \\
\hline NSR & 2 & 0,9 & 0 & 0,0 & 2 & 0,6 & \\
\hline \multicolumn{8}{|c|}{ Quem pode utilizar a vacina anti HPV? } \\
\hline Mulheres & 60 & 28,2 & 33 & 27,7 & 93 & 28,0 & \multirow{3}{*}{0,972} \\
\hline Ambos & 150 & 70,4 & 86 & 72,3 & 236 & 71,1 & \\
\hline NSR & 3 & 1,4 & 0 & 0,0 & 3 & 0,9 & \\
\hline
\end{tabular}

Para que faixa etária a vacina anti HPV é recomendada?

\begin{tabular}{c|c|c|c|c|c|c|c}
\hline 10 a 20 anos & 205 & 96,2 & 98 & 82,4 & 303 & 91,3 & \\
\cline { 1 - 6 } 21 a 30 anos & 7 & 3,3 & 20 & 16,8 & 27 & 8,1 & $<0.0001^{*}$ \\
\hline 31 a 40 anos & 1 & 0,5 & 1 & 0,8 & 2 & 0,6 & \\
\hline
\end{tabular}

As mulheres vacinadas precisam realizar o exame Papanicolau anualmente?

\begin{tabular}{c|c|c|c|c|c|c|c}
\hline Sim & 193 & 90,6 & 109 & 91,6 & 302 & 91,0 & \multirow{2}{*}{0,920} \\
\cline { 1 - 6 } Não & 20 & 9,4 & 10 & 8,4 & 30 & 9,0 & \\
\hline
\end{tabular}

Adicionalmente, em outros estudos também houve concordância $(75,6 \%)$ do papel da prevenção do câncer garantido pela vacinação ${ }^{22}$, no entanto por se tratar de um tema relativamente novo seriam necessários melhores esclarecimentos para a comunidade acadêmica sobre a vacina ${ }^{23}$.

0 desempenho nas questões relativas à vacina foi bastante heterogêneo em estudos semelhantes. Nas Universidades Kebangsaan da Malásia e Malaya (UKM e UM, respectivamente) ${ }^{15}$, os estudantes se revelaram portadores de conhecimentos mais amplos sobre a vacina. Em outro estudo, realizado na Universidade de Atenas, as menores taxas de respostas corretas foram relacionadas às indicações e idade adequada para a vacinação, além do fato de que a avaliação global, dos estudantes gregos, ser considerada abaixo dos níveis desejados ${ }^{24}$. 
É fundamental um nível de conhecimento apropriado acerca da vacina. Em pesquisas realizadas com jovens do ensino médio e universitários houve baixo conhecimento da existência da vacina como opção para prevenir a infecção pelo HPV (22,3\% dos jovens sabiam da vacina), além de baixos escores (20,0\% de respostas corretas) no conhecimento global sobre o vírus ${ }^{25}$.

A pesquisa com os acadêmicos da PUC-Goiás não levou em consideração a possibilidade de interferência de fatores sociais, determinantes na aquisição de diferentes conhecimentos prévios. Entretanto, estudos semelhantes buscaram relacionar fatores geográficos, étnicos e comportamentais ao conhecimento acerca do HPV e a vacina. Um estudo $^{26}$, realizado entre universitários negros na Flórida, provou que, apesar de $64,0 \%$ dos 351 estudantes terem ouvido falar do HPV, não havia diferença significante em relação às amostras da população universitária em geral.

Em uma pesquisa feita com 1109 universitários, da Universidade do Alabama (EUA), percebeu-se uma associação de diversos fatores aos baixos escores de pontuação (índice de acerto por volta de 50\%) no questionário sobre 0 papilomavírus, como não ter água corrente, não ter seguro de saúde e ser do sexo masculino ${ }^{21}$. Em contrapartida, em instituições de ensino superior (universidades e institutos tecnológicos) em Atenas (Grécia) ${ }^{24}$, conseguiu relacionar melhor desempenho nos testes aplicados entre universitários na faixa de 21 a 26 anos, já vacinados contra o HPV, não solteiros e com algum familiar e/ou amigo com infecção por HPV.

Avaliando as médias de acerto de acordo com o avançar de cada semestre, percebe-se que houve uma melhora no desempenho das turmas com o avançar no curso de Medicina, tendo uma variação de acerto de 71,7\% (na turma do primeiro semestre) até $83,2 \%$ da turma do décimo segundo semestre.

No que diz respeito ao desempenho geral dos acadêmicos da PUC-Goiás, a porcentagem geral de acerto foi de 80,0\%, superior a todos os outros estudos ${ }^{19,22,27,28,29}$, metodologicamente comparáveis, identificados na literatura especializada.

Avaliando o desempenho de acordo com o grupo de conhecimentos, verifica-se um comprometimento maior das questões relacionadas aos sinais e sintomas e diagnóstico do câncer de colo uterino. Dessa forma, destacamos a importância de solucionar as dúvidas acerca desse tema.

Pode-se perceber que o desempenho do gênero feminino se manteve superior ao do masculino em quase todas as turmas. Entre todos os participantes, a média de acerto foi de $78,3 \%$ e $80,9 \%$, para os gêneros masculino e feminino, respectivamente. Em um estudo realizado em Portuga ${ }^{30}$, as participantes do sexo feminino apresentaram um melhor desempenho no conhecimento sobre o HPV, câncer cervical e a vacina, ainda que o desempenho geral não tenha sido o desejado.

Diversos estudos foram feitos somente com mulheres para avaliar o conhecimento sobre o HPV, a vacina e a aceitação da vacinação por parte delas ${ }^{31}$. Em um estudo realizado em Pequim na China ${ }^{20}$ percebeu-se que 0 nível de conhecimento dentre as estudantes universitárias foi inferior aos das mulheres fora da universidade, porém, após intervenção educativa com os dois grupos, as universitárias tiveram um melhor desempenho geral em um questionário aplicado após intervenção. Estudos semelhantes apresentam resultados heterogêneos. Enquanto um estudo32 obteve um nível de conhecimento significativo com 305 acadêmicos na Universidade Kebangsaan da Malásia e Universidade Malaya, nos demais estudos não conseguiram uma conclusão tão esperançosa $a^{19,22,27,28,29}$.

Na Universidade de Chongqing (China) a média de acerto dentre os 878 estudantes de Medicina participantes foi $45,4 \%$, apesar do conhecimento melhorar com o tempo de estudo na Universidade ${ }^{19}$. Na Índia, os próprios acadêmicos reconheciam as falhas no conhecimento sobre o HPV e sua vacina, por ser um tema relativamente novo, segundo o autor ${ }^{22}$.

Observam-se, nos estudos semelhantes anteriores a este, que alguns realizam apenas a avaliação do conhecimento dos universitários, porém, determinados estudos promoveram trabalhos de educação com os acadêmicos baseados no pré-teste aplicado ${ }^{27}$. Com os resultados das avaliações após a intervenção, houve melhora significante no desempenho ${ }^{33}$. Outros estudos também fizeram uma intervenção e avaliação pós-teste, sendo a longo prazo, e obtiveram desempenhos não tão animadores ${ }^{34}$. Alguns destes, destacaram a necessidade de campanhas frequentes para manter a efetividade a longo prazo das interferências educativas ${ }^{27}$.

Dentre as limitações do estudo, destacam-se a baixa adesão por parte dos acadêmicos (taxa de participação de $60,9 \%$ ), falta de intervenções educativas, bem como a investigação mais detalhada dos acadêmicos sobre determinados fatores culturais investigados em outros estudos semelhantes. 


\section{Considerações Finais}

A partir do estudo com os acadêmicos de Medicina da PUC-Goiás, pode-se perceber que o desempenho nos instrumentos de avaliação do conhecimento acerca do vírus HPV, o câncer de colo uterino e a vacina contra o HPV foram satisfatórios, quando comparado com outros estudos semelhantes, tendo poucos pontos de dúvidas, notadamente em relação ao conhecimento sobre o câncer cervical.

Adicionalmente, percebe-se que, com o decorrer da formação no curso de Medicina, houve uma progressão no conhecimento até o último semestre. Este com um escore de acerto suficiente para informar e sanar as principais dúvidas da população. Vislumbram-se grandes possibilidades de continuação e aprofundamento da pesquisa, baseado no enfoque feito em outros estudos de temática semelhante.

\section{Referências}

1. Panobianco MS, Lima ADF, Oliveira ISB, Gozzo TO. Knowledge concerning HPV among adolescent undergraduate nursing students. Texto Contexto Enferm. [online]. 2013; 22(1):201-207.

2. Leto MGP, Santos Junior GF, Porro AM, Tomimori J. Human papillomavirus infection: etiopathogenesis, molecular biology and clinical manifestations. An. Bras. Dermatol. [online]. 2011; 86(2):306-317.

3. Oliveira MC, Soares RC, Pinto LP, CostaALL. HPV e carcinogênese oral: revisão bibliográfica. Rev. Bras. Otorrinolaringol. [online]. 2003; 69(4):553-559.

4. Nakagawa JTT, Schirmer J, Barbieri M. Vírus HPV e câncer de colo de útero. Rev. Bras. Enferm. [online]. 2010; 63(2):307-311.

5. Brasil. Vacina contra o HPV Prevenção contra câncer de colo do útero no SUS e inovação tecnológica para o Brasil. Ministério da Saúde, 2013

6. Federação Brasileira das Associações de Ginecologia e Obstetrícia, Sociedade Brasileira de Cancerologia. Câncer do Colo Uterino: Tratamento. Diretrizes Clínicas na Saúde Suplementar. 2011.

7. Alves DB, Tozetti IA, Gatto Flávia A, Cassandri F, Ferreira AMT, Carlos ESF et al. Linfócitos CD4, CD8 e células NK no estroma da cérvice uterina de mulheres infectadas pelo papilomavírus humano. Rev. Soc. Bras. Med. Trop. 2010; 43(4):425-429.

8. Ayres ARG, Silva GA. Cervical HPV infection in Brazil: systematic review. Rev. Saúde Pública [online]. 2010; 44(5):963-974.

9. Saslow D, Castle PE, Cox JT, Davey DD, Einstein MH, Ferris DG, Goldie SJ, Harper DM, Kinney W, Moscicki AB, Noller KL, Wheeler CM, Ades T, Andrews KS, Doroshenk MK, Kahn KG, Schmidt C, Shafey O, Smith RA, Partridge EE; Gynecologic Cancer Advisory Group, Garcia F. American Cancer Society Guideline for human papillomavirus (HPV) vaccine use to prevent cervical cancer and its precursors. CA Cancer J Clin. 2007; 57(1):7-28.

10. Borsatto AZ, Vidal MLB, Rocha RCNP. Vacina contra o HPV e a Prevenção do Câncer do Colo do Útero: Subsídios para a Prática. Revista Brasileira de Cancerologia 2011; 57(1):67-74.

11. Carvalho NS, Kannemberg AP, Munareto C, Yoshioka D, Absy MCV, Ferreira MA, Tambara Filho R. Associação entre HPV e câncer peniano: revisão de literatura. J Bras Doenças Sex Transm. 2007; 19(2):92-5.

12. Giraldo PC, Silva MJPMA, Fedrizzi EM, Gonçalves AKS, Amaral RLG, Eleutério Junior J, Figueiredo IV.Prevenção da infecção por HPV e lesões associadas com o uso de vacinas. J Bras Doenças Sex Transm. 2008; 20(2):132-140.

13. Herrera-Ortiz A, Arriaga-Demeza CR, Conde-González CJ, Sánchez-Alemán MA. Knowledge about herpes simplex virus type 2 and human papillomavirus, and risk perception to acquire infections among college students. Gac Med Mex. 2013; 149(1):16-26.

14. Nadal SR, Manzione CR. Rastreamento e seguimento dos portadores das lesões anais induzidas pelo papilomavírus humano como prevenção do carcinoma anal. Rev bras. colo-proctol. [online]. 2009; 29(2):250-253.

15. Rashwan HH, Saat NZ, Abd Manan DN. Knowledge, attitude and practice of malaysian medical and pharmacy students towards human papillomavirus vaccination. Asian Pac J Cancer Prev. 2012; 13(5):2279-2283.

16. Yacobi E, Tennant C, Ferrante J, Pal N, Roetzheim R. University students' knowledge and awareness of HPV. Prev Med. 1999; 28(6):535-41.

17. llozumba UO. HPV vaccination knowledge and attitudes amongst students in two and four year colleges in Atlanta, Georgia. [Thesis]. Faculty of the Rollins School of Public Health of Emory University. 2011; 49p. 
18. Pereira MG. Epidemiologia: teoria e prática. Rio de Janeiro: Guanabara-Koogan, 1995.

19. Fu CJ, Pan XF, Zhao ZM, Saheb-Kashaf M, Chen F, Wen Y, Yang CX, Zhong XN. Knowledge, perceptions and acceptability of HPV vaccination among medical students in Chongqing, China. Asian Pac J Cancer Prev. 2014; 15(15):6187-93.

20. Chang IJ, Huang R, He W, Zhang SK, Wang SM, Zhao FH, Smith JS, Qiao YL. Effect of an educational intervention on HPV knowledge and vaccine attitudes among urban employed women and female undergraduate students in China: a cross-sectional study. BMC Public Health. 2013; 13:916.

21. Vogtmann E, Harlow SD, Valdez AC, Valdez JC, Ponce EL. HPV knowledge in Mexican college students: implications for intervention programmes. Health Soc Care Community. 2011; 19(2):148-57.

22. Pandey D, Vanya V, Bhagat S, VS B, Shetty J. Awareness and Attitude towards Human Papillomavirus (HPV) Vaccine among Medical Students in a Premier Medical School in India. PLoS ONE. 2012; 7(7): e40619.

23. Carvalho NS, Teixeira LM, Pradel EM, Gabardo J, Joly C, Urbanetz AA. Vaccinating against HPV: physicians' and medical students' point of view. Vaccine. 2009; 27(20):2637-40.

24. Donadiki EM, Jiménez-García R, Hernández-Barrera V, Carrasco-Garrido P, López de Andrés A, Jimenez-Trujillo I, Velonakis EG. Knowledge of the HPV vaccine and its association with vaccine uptake among female higher-education students in Greece. Hum Vaccin Immunother. 2013; 9(2):300-5.

25. Markham CM, Escobar-Chaves SL, Addy RC, Lewis H, Tortolero-Luna G, Tortolero SR: Knowledge and risk perception regarding HPV among Latino alternative school students in Houston, Texas. Tipica. 2009, 5(1):32-38.

26. D'Urso J, Thompson-Robinson M, Chandler S. HPV knowledge and behaviors of black college students at a historically black university. J Am Coll Health. 2007; 56(2):159-63.

27. S. M. McCusker, I. Macqueen, G. Lough, A. I. MacDonald, C. Campbell, and S. V. Graham, "Gaps in detailed knowledge of human papillomavirus (HPV) and the HPV vaccine among medical students in Scotland," BMC Public Health. 2013; 13:264. 28. Wen Y, Pan XF, Zhao ZM, Chen F, Fu CJ, Li SQ, Zhao Y, Chang H, Xue QP, Yang CX. Knowledge of human papillomavirus (HPV) infection, cervical cancer, and HPV vaccine and its correlates among medical students in Southwest China: a multi-center cross-sectional survey. Asian Pac J Cancer Prev. 2014; 15(14):5773-9.

29. Ghojazadeh M, Azar ZF, Saleh P, Naghavi-Behzad M, Azar NG. Knowledge and attitude of Iranian University students toward human papilloma virus. Asian Pac J Cancer Prev. 2012; 13(12):6115-9.

30. Medeiros R, Ramada D. Knowledge differences between male and female university students about human papillomavirus (HPV) and cervical cancer: Implications for health strategies and vaccination. Vaccine. 2010; 29(2):153-60. 31. Wang HH, Wu SY. HPV vaccine knowledge and perceived risk of cervical cancer among female college students in Taiwan. Asian Pac J Cancer Prev. 2013; 14(12):7371-4.

32. Rashwan H, Lubis SH, Ni KA. Knowledge of cervical cancer and acceptance of HPV vaccination among secondary school students in Sarawak, Malaysia. Asian Pac J Cancer Prev. 2011; 12(7):1837-41.

33. Merzouk MD, Courtney P, Garrett-Albaugh S, Janoo J, Hobbs G, Vernon M. Knowledge of HPV in West Virginia high school health students and the effects of an educational tool. J Pediatr Adolesc Gynecol. 2011; 24(5):278-81.

34. Yanikkerem E, Piyan G, Kavlak T, Karadeniz G. Assessing the role of education on Turkish university students' knowledge about HPV and related diseases. Asian Pac J Cancer Prev. 2010; 11(6):1703-11. 


\section{Samuel Laurindo da Silva}

Endereço para correspondência - Av. Universitária n. 1440, Área 4, Bloco K - Setor Universitário.

CEP: 74605-010, Goiânia, GO, Brasil.

E-mail: samuellsilva@gmail.com

Lattes: http://lattes.cnpq.br/9842132561654796

Alfredo Lustosa Vargas - alfredolvargas@gmail.com

Rogério José de Almeida - rogeriopucgo@gmail.com

Vera Aparecida Saddi - verasaddi@gmail.com

Jacqueline Andréia Bernardes Leão Cordeiro - jackbl@uol.com.br

Antonio Márcio Teodoro Cordeiro Silva - marciocmed@gmail.com

Enviado em 26 de abril de 2017.

Aceito em 21 de agosto de 2017. 